\title{
Protocol Amendment
}

National Cancer Institute

\section{Source}

National Cancer Institute. Protocol Amendment. NCI Thesaurus. Code C132347.

A written description of a change(s) to, or formal clarification of, a protocol. (ICH E6) 